\title{
Spontaneous and Persistent Currents in Mesoscopic Aharonov-Bohm Loops: Static Properties and Coherent Dynamic Behavior in Crossed Electric and Magnetic Fields ${ }^{\text {II }}$
}

\author{
I. O. Kulik \\ Department of Physics and Astronomy, Stony Brook University, \\ Stony Brook, NY, 11794-3800 USA \\ Department of Physics, Bilkent University, Ankara, 06533 Turkey \\ e-mail:iokulik@yahoo.com \\ Received June 8, 2004
}

\begin{abstract}
Mesoscopic or macromolecular conducting rings with a fixed number of electrons are shown to support persistent currents due to the Aharonov-Bohm flux, and the "spontaneous" persistent currents without the flux when structural transformation in the ring is blocked by strong coupling to the externally azimuthal-symmetric environment. In the free-standing macromolecular ring, symmetry breaking removes the azimuthal periodicity, which is further restored at the increasing field, however. The dynamics of the Aharonov-Bohm loop in crossed electric and magnetic fields is investigated within the tight-binding approximation; we show that transitions between discrete quantum states occur when static voltage pulses of prescribed duration are applied to the loop. In particular, the three-site ring with one or three electrons is an interesting quantum system that can serve as a qubit (quantum bit of information) and a qugate (quantum logical gate) because in the presence of an externally applied static electric field perpendicular to a magnetic field, the macromolecular ring switches between degenerate ground states mimicking the NOT and Hadamard gates of quantum computers. (C) 2005 Pleiades Publishing, Inc.
\end{abstract}

\section{PERSISTENT CURRENTS IN MESOSCOPIC SYSTEMS}

Persistent currents have been predicted for mesoscopic conducting ballistic or quasiballistic loops $\left([1]^{1}\right.$ and references therein, [2]) that do not show the effect of superconductivity and that have been extended to diffusive rings [3]. The current appears in the presence of a magnetic field as a result of the Aharonov-Bohm effect [4], demonstrating the special role of the vector potential in quantum mechanics. As discussed in [5], persistent currents are similar to orbital currents in normal metals first considered by Teller [6] in his interpretation of Landau diamagnetism in metals [7], but are specific to the doubly connected geometry of conductors (loops, hollow cylinders, etc.). Persistent currents have been observed in indirect $[8,9]$ as well as direct $[10,11]$ experiments, showing the single-flux-quantum $\Phi_{0}=$ hcle periodicity in the resistance of thin $\mathrm{Nb}$ wires [8] and networks of isolated $\mathrm{Cu}$ rings [9], and in singleloop experiments on metals [10] and semiconductors [11]. In [12], the periodic variation of resistivity in

\footnotetext{
II The text was submitted by the author in English.

${ }^{1}$ This paper proved exact periodicity of ring energy as a function of the magnetic flux with the period hcle, although with an indefinite amplitude.
}

molecular conducting cylinders (carbon nanotubes) was attributed to the Altshuler-Aronov-Spivak effect [13], a companion to the classical Aharonov-Bohm mechanism with the twice smaller periodicity in magnetic flux $\Phi_{1}=h c / 2 e$. A further trend in macromolecular persistent currents [14-16] is in the quantum computational [17] prospects of using the Aharonov-Bohm loops as qubits with an advantage of easier (radiationfree) manipulation of qubit states, and in the increased decoherence times compared to macroscopic "Schrödinger cat" structures (Josephson junctions).

The present paper focuses on ballistic AharonovBohm rings, like those naturally found in molecular crystals with metalloorganic complexes as the building blocks $[18,19]$. We approximate such macromolecular structures as rings with resonant hopping of electrons between the near-site atoms or complexes serving as electron localization sites. As shown in [14], the smallest (three-site) persistent current ring displays a $\Lambda$-shaped energy configuration (Fig. 1) with two degenerate ground states, at the external flux through the ring equal to half the normal-metal flux quantum, $\Phi=h c / 2 e$. At a certain number of electrons in the ring, persistent current appears at zero field (the "spontaneous" current). The spontaneous persistent current loop, to be discussed below, achieves the degenerate state at zero 


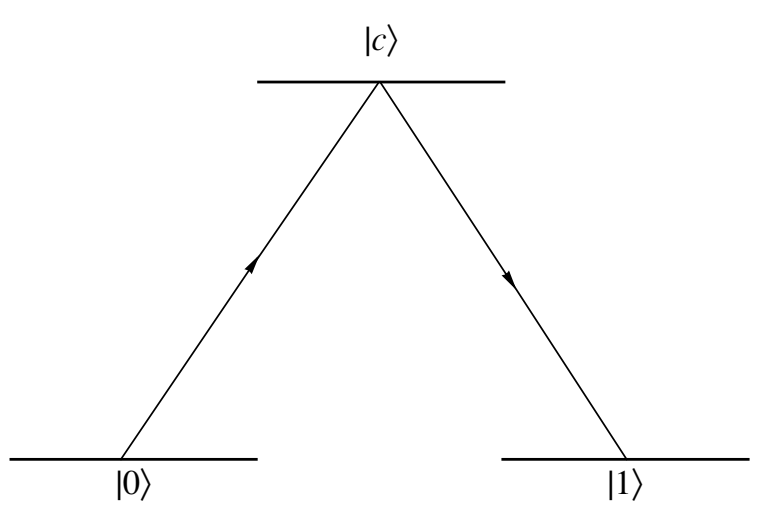

Fig. 1. A $\Lambda$-shaped energy configuration in the AharonovBohm ring. Arrows indicate a transition between degenerate states $|0\rangle$ and $|1\rangle$ through virtual transition to the control state $|c\rangle$.

(a)
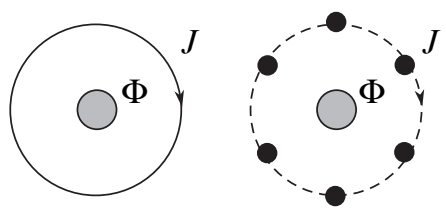

(b)

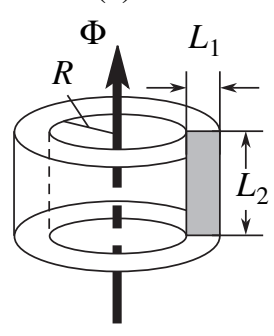

Fig. 2. (a) Models of mesoscopic and nanoscopic Aharonov-Bohm loops: a one-dimensional continuous loop; (b) a discrete loop with regularly spaced centers of electron localization (sites); (c) a 3-dimensional loop in the form of a cylinder with a longitudinal dimension of $L=2 \pi R$ and transverse dimensions of $L_{1}, L_{2}$.

field or, if the degeneracy is lifted by the electronphonon coupling, at reasonably small fields.

Persistent current is a voltage-free nondecaying current that exists as a manifestation of the fact that the ground state of a doubly connected conductor in a magnetic field is a current-carrying one. This statement was proved for ballistic loops [2] and for diffusive rings [3]. There is no principal difference between these extremes. Counterintuitively, a ballistic structure does not show infinite conductivity, as was sometimes naively supposed; a dc resistance of the loop is infinite rather than zero when a dc electric field is applied to the system. In the case where a current is fed through the structure, no voltage appears provided the magnitude of the current is smaller than a certain critical value. This applies to both elastic and inelastic scatterings. The magnitude of the critical current of a ballistic ring smoothly matches the current of the diffusive ring when the mean free path of the electron becomes large. In a dirty limit, $l \ll L$, where $l$ is the electron mean free path and $L$ is the ring circumference, the critical value of the persistent current decreases proportionally to $l / L$ according to [20], or to $(l / L)^{1 / 2}$ according to numerical simulation [5]. The nondecaying current does not even require severe restrictions on the so-called "phase breaking" mean free path $l_{\varphi}$. In fact, the normal-metal supercurrent is an analogue of the "nonquantum" Josephson effect [21, 22], the one in which the phase of superconductor is considered a classical variable. Stronger criteria (the dephasing length larger than the system size, and the analogous requirement in the time domain, that the "decoherence time" is larger than the characteristic time of observation) apply to persistent current rings as quantum computational tools, which are analogs of macroscopic quantum tunneling [2326]. Persistent current is a thermodynamic property, clearly distinct from the dissipative currents in conductors, and can in principle exist in a system that has the vanishing Ohmic conductance.

\section{SPONTANEOUS PERSISTENT CURRENTS}

Persistent current in a ballistic ring appears due to the Aharonov-Bohm flux. The current, however, can also occur when the external magnetic field is zero, in which case it is called the spontaneous current. Such a situation was noticed accidentally by various authors, in particular, [27, 28], but did not seem convincing, did not attract attention due to fixed chemical potential configuration studied, and was attributed to the effect of Peierls instability in the ring [29-32] (with the latter paper criticized $[33,34]$ in regard to the inaccuracy of the mean-field approximation). In fact, the fixed-number-of-particle ring with an odd number of electrons displays a number of structural instabilities, of which the Peierls transformation [35] and the Jahn-Teller effect [36] are the best known examples, or generally, a more complex atom rearrangement when the ground state proves degenerate in a symmetric configuration.

The origin of the spontaneous current can be understood as follows. We consider a one-dimensional ring in the field of a vector potential created by a thin, infinitely long solenoid perpendicular to the plane of the ring and piercing the ring (Fig. 2a). The electron energy in the ring is

$$
\varepsilon_{n}=\frac{h^{2}}{2 m L^{2}}\left(n-\frac{L A}{\Phi_{0}}\right)^{2}
$$

where $A=\Phi / L$ is the angular component of the vector potential ( $\Phi$ is the total magnetic flux of the solenoid) and $n=0, \pm 1, \pm 2, \ldots$. Such a state corresponds to the current

$$
J=-\frac{c \partial \varepsilon_{n}}{\partial \Phi}=\frac{e h}{m L^{2}}\left(n-\frac{\Phi}{\Phi_{0}}\right)
$$

which is zero at $\Phi=0$ and $n=0$, but is nonzero at $n \neq 0$ even at zero flux. At $T=0$, electrons, in the total number $N$, occupy the lowest possible energies compatible with 
the Pauli exclusion principle, i.e., such that each state is occupied with two electrons with opposite spins at most. Therefore, the ground state of one or two electrons is that of $n=0$, and hence has zero current at $\Phi=0$. But the state with the next electron number, $N=3$, already resumes at $n=1$ or $n=-1$, or is in a superposition of these states, $\alpha|1\rangle+\beta|-1\rangle$, depending on the way the state at the initial condition is prepared, and therefore carries a current unless $\alpha \neq \beta$. If there is no decoherence of the state due to the interaction of the loop with the environment, the current persists in time without any voltage applied along the loop. This applies to a ballistic perfectly symmetric ring. The inhomogeneity in the ring, as well as scattering of electrons by impurities, may result in a nondegenerate current-free state. This is illustrated in Fig. 3 for the ring with a $\delta$-functional barrier $V_{0} \delta(x)$, which results in the Kronig-Penney equation for energy,

$$
\cos (2 \pi k)+\frac{V_{0} L}{2 \varepsilon_{0}} \frac{\sin (2 \pi k)}{2 \pi k}=\cos \left(2 \pi \frac{\Phi}{\Phi_{0}}\right) .
$$

The electron energy is $\varepsilon=\varepsilon_{0} k^{2}$, where $k=k_{n}$ is one of solutions to Eq. (2) and $\varepsilon_{0}=h^{2} / 2 m L^{2}$. The same conclusion is obtained for a discrete Aharonov-Bohm ring (Fig. 2b), to be considered in detail below.

Figure 4 shows the maximum value of persistent current, as well as that of the spontaneous current introduced above, versus the number of electrons in a threedimensional ballistic ring (the one with the electron mean free path $l=\infty$ ) modeled as a finite-length hollow cylinder (Fig. 2c) with the rectangular cross section $L_{1} \times L_{2}$ containing a finite number of perpendicular electron channels

$$
N_{\perp}=\frac{L_{1} L_{2} k_{\mathrm{F}}^{2}}{2 \pi^{2}} .
$$

We note that the magnitude of the current in a ballistic ring is not $e v_{\mathrm{F}} / L$, as is sometimes suggested ( $v_{\mathrm{F}}$ is the Fermi velocity), but

$$
J_{\max } \sim \frac{e V_{\mathrm{F}}}{L} N_{\perp}^{1 / 2}
$$

(see also [2]). The dependence $J_{\max }(N)$ at $T=0$ is irregular due to the contribution to the total current of both the negative and positive terms originating from different electron eigenstates.

Figure 5 explains the origin of persistent current as a bistability effect in a ring. While the electron energy has a minimum at $\Phi=0$ for an even number of electrons, it acquires a maximum when the number of electrons is odd. The inductive energy, to be included below, shifts the position of minima in that curve only very slightly. The spontaneous current has the same order of magnitude as the maximum persistent current,

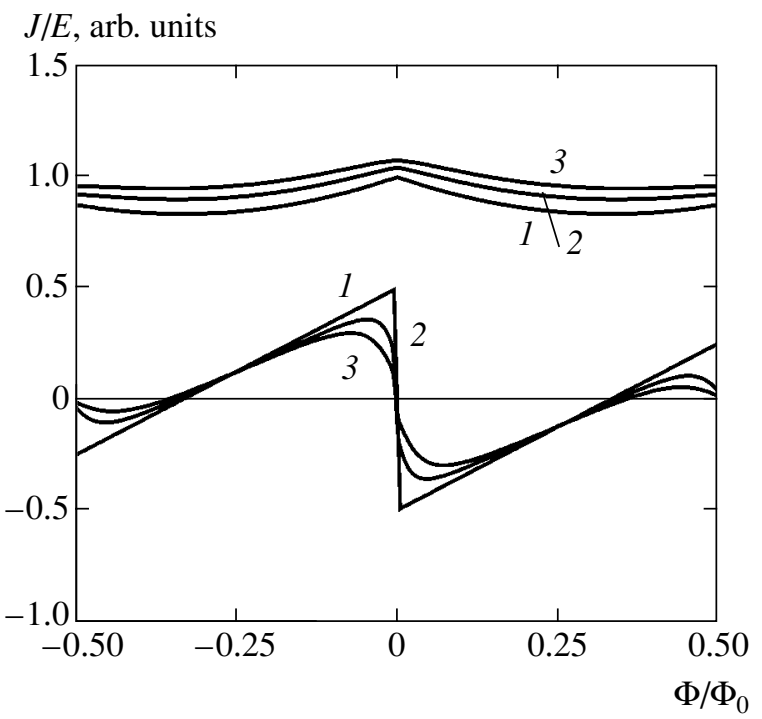

Fig. 3. Ground state energies and currents in the continuous ring with 3 electrons at various strengths of the barrier: $g=$ 0 (1), 1 (2), 2 (3).

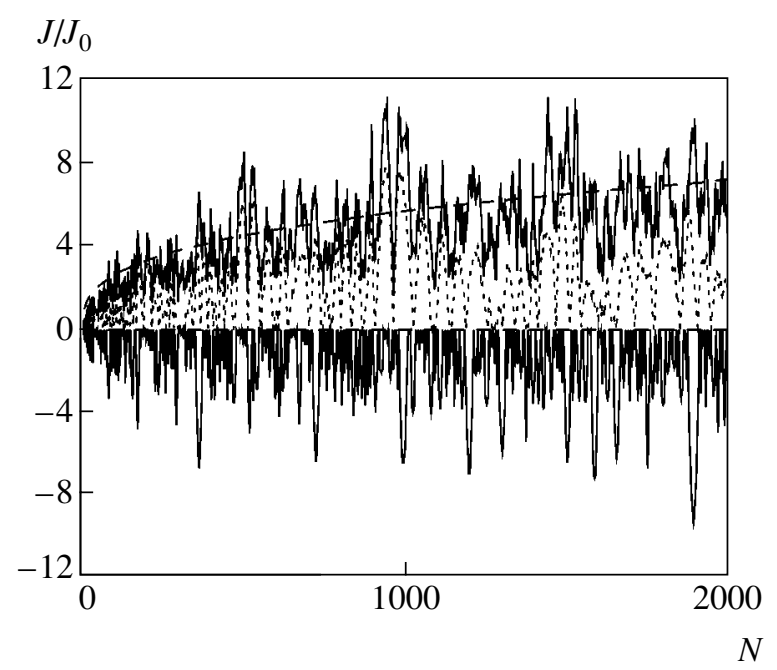

Fig. 4. Persistent current versus the number of electrons in a ring with ratio cross-sectional dimensions $L: L_{1}: L_{2}=$ $10: 1: 1$ (configuration with spin). The upper curve is the maximum current in units of $J_{0}=e v_{\mathrm{F}} / L$ at given $N$; the dotted curve is the amplitude of the first harmonic of $J_{\text {pers }}(\Phi)$; and the curve at negative $J$ is the spontaneous persistent current, also in units of $J_{0}$. The dashed curve is the square root of the number of perpendicular channels $N_{\perp}$.

and it is an inseparable part of the Aharonov-Bohm effect in a ballistic ring.

In a one-dimensional loop, discrete quantum states are

$$
\psi_{n}=\frac{1}{\sqrt{L}} e^{\mathrm{in} \theta}
$$




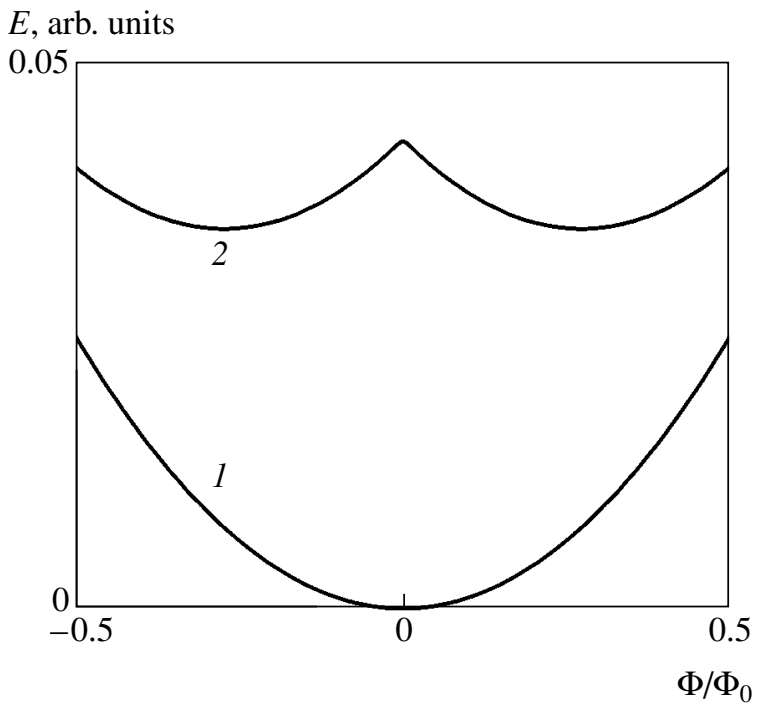

Fig. 5. Examples of the occurrence of a bistable configuration in a ring. Energy versus flux in a ring of $10(1)$ and 11 (2) electrons. Curve 2 is shifted downward for convenience but is not reset.

where $\theta$ is the azimuthal angle, with the energies given by (1) plus the inductive energy of the current. For the loop with three electrons, this gives the total energy,

$$
E(f)=\varepsilon_{0}\left[f^{2}+\frac{1}{2}( \pm 1-f)^{2}\right]+\frac{\mathscr{L} J_{0}^{2}}{2 c^{2}} j^{2}(f),
$$

corresponding at $\Phi=0$ to two spin-1/2 states with $n=$ 0 and one state with $n=1$ or $n=-1$. The last term in Eq. (4) is the magnetic inductive energy and $\mathscr{L}$ is the inductance (of the order of the ring circumference, in the units adopted). The current

$$
J=-\frac{e}{h} \frac{\partial E}{\partial f}
$$

is equal to

$$
J(f)=J_{0}( \pm 1-3|f|), \quad J_{0}=\frac{e \varepsilon_{0}}{h}
$$

and is nonzero at $f=0$ in either of the states \pm . The ratio of the magnetic energy to the kinetic energy is on the order of

$$
\eta=\frac{\mathscr{L} J_{0}^{2}}{2 c^{2} \varepsilon_{0}} \approx \frac{e^{2}}{4 \pi m c^{2} R} \sim 10^{-6} \frac{a_{0}}{R},
$$

where $a_{0}$ is the Bohr radius. This is a very small quantity, and therefore the magnetic energy is unimportant in the energy balance of the loop. The total flux in the loop is $f=f_{\text {ext }}+2 \eta j(f)$, where $f_{\text {ext }}$ is the external flux and $j(f)=J(f) / J_{0}$. The correction to the externally applied flux is significant only at very small fields $f_{\text {ext }} \sim \eta$; otherwise we can ignore this contribution.

When a persistent-current loop is placed in an electric field perpendicular to a magnetic field, the system coherently switches between the discreet states of the loop providing for quantum transitions (quantum logical gates) in the loop performing as a qubit in a quantum computer. This aspect of persistent currents in ballistic loops is analyzed in Section 3.

The property of a nonzero spontaneous persistent current thus demonstrated for noninteracting electrons survives strong electron-electron coupling but collapses when the coupling to the lattice is included. This is considered in detail in Section 4. In what follows, the structural transformation in the ballistic ring is investigated in an exact way by considering the ring dynamics in the tight-binding approximation [37, 38]. The "lattice" (the atomic configuration of the loop) can respond to the bistable state by a readjustment of atoms similar to the Peierls transition (doubling of the lattice period in a one-dimensional atomic chain, see, e.g., [39, 40]), or by a more general lattice transformation that does not reduce to simple doubling. When the loop is in the rigid background in the periodic lattice on a substrate of a much stronger bound solid, the degeneracy may not be lifted, or may remain in a very narrow interval of the externally applied field.

\section{DYNAMICS OF PERSISTENT CURRENTS IN CROSSED ELECTRIC AND MAGNETIC FIELDS}

The Hamiltonian of the ring consisting of $N$ sites localizing electrons at equidistant angular positions is $\theta_{n}=2 \pi n / N$ is

$$
H_{0}=-\tau \sum_{n=1}^{N}\left(a_{n}^{+} a_{n+1} e^{i \alpha}+a_{n+1}^{+} a_{n} e^{-i \alpha}\right),
$$

where $a_{n}^{+}$is a fermionic operator creating (and $a_{n}$, annihilating) the electron at the site $\mathbf{R}_{n}$ in the ring with the periodic boundary condition $a_{N+1}=a_{1}$, and $\alpha=$ $2 \pi \Phi / N \Phi_{0}$ is the phase related to the Aharonov-Bohm flux threading the ring. Placing the ring in the homogeneous electric field perpendicular to the magnetic field (Fig. 6) results in the extra term

$$
H_{1}=V_{0} \sum_{n=1}^{N} \cos \frac{2 \pi n}{N} a_{n}^{+} a_{n}
$$

being added to the Hamiltonian. The Hamiltonian $H_{0}$ is diagonalized by the angular momentum (i.e., $m=0,1$, 
$\ldots, N-1)$ eigenstates $A_{m}^{+}|0\rangle$ such that

$$
A_{m}^{+}=\frac{1}{\sqrt{N}} \sum_{n=0}^{N-1} a_{n}^{+} \exp \frac{2 \pi i m n}{N} .
$$

These states have the energies

$$
\varepsilon_{m}=-2 \tau \cos \frac{2 \pi}{N}\left(m-\frac{\Phi}{\Phi_{0}}\right)
$$

plotted versus the flux in Fig. 7. The electronic configuration at $\Phi=\Phi_{0} / 2$ has a $\Lambda$-shaped energy structure with two degenerate ground states shown in Fig. 1, which were suggested as $|0\rangle$ and $|1\rangle$ components of a qubit in $[14,15]$. The time evolution of angularmomentum eigenstates $A_{m}^{+}|0\rangle$ is periodic at certain values of $V_{0}$ and at the value of the flux equal to half the flux quantum $\Phi_{0} / 2=h c / 2 e$.

In the eigenbasis of the operators $A_{m}$, the Hamiltonian $H_{0}+H_{1}$ at $N=3$ in the absence of an electric field is transformed into the diagonal form (we scale all energies in units of $\tau$ )

$$
H_{0}=\sum_{m} \varepsilon_{m} A_{m}^{+} A_{m}=\left(\begin{array}{ccc}
-1 & 0 & 0 \\
0 & 2 & 0 \\
0 & 0 & -1
\end{array}\right)
$$

and the Hamiltonian $H_{1}$ becomes

$$
H_{1}=\left(\begin{array}{ccc}
0 & V & V \\
V & 0 & V \\
V & V & 0
\end{array}\right),
$$

where $v=V_{0} / 2 \tau$. We let the $m=1$ and $m=3$ states be denoted by $|0\rangle$ and $|1\rangle$, in the qubit terminology, and the excited state $m=2$ by $|c\rangle$ (the "control" state coupling qubit states to the "qugate," or the quantum logic gate).

The eigenstates of $H_{0}+H_{1}$ versus $v$ at $\Phi=\Phi_{0} / 2$ are presented in Fig. 8. We assume that at $t \leq 0$, the potential is $V_{0}=0$, such that the system at $t=0$ is a superposition of the angular momentum states $A_{m}^{+}|0\rangle$ with certain amplitudes $C_{m}(0)$. At a later time and at a constant value of $V_{0}, C_{n}(t)$ evolves as

$$
C_{n}(t)=\sum_{m} \exp \left(-i\left(H_{0}+H_{1}\right) t\right)_{m n} C_{m}(0)
$$

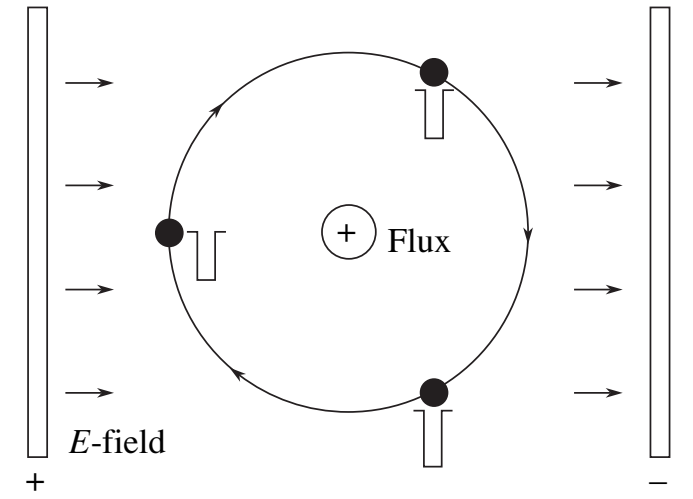

Fig. 6. Scheme of a 3-site qubit in the electric field perpendicular to the magnetic field.

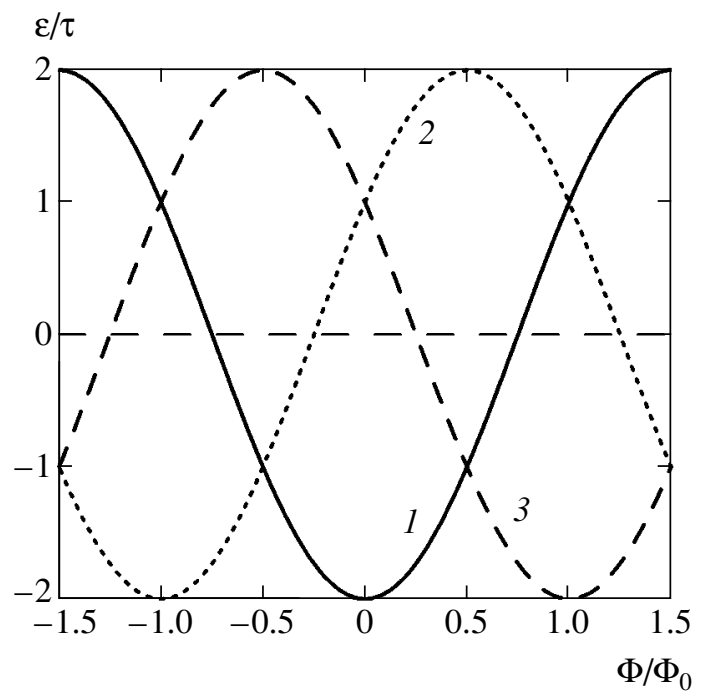

Fig. 7. Curves 1 and 3 are energy versus magnetic flux dependences in the degenerate states carrying opposite currents. The current is found as the derivative $j=-c \partial \varepsilon / \partial \Phi$. Curve 2 corresponds to the zero-current virtual state at the operating point of a qubit at the half-flux quantum $\Phi=$ $\Phi_{0} / 2$.

For a step function $V(t)=V_{0} \theta(t)$, this gives the dependence [14]

$$
C_{n}(t)=\sum_{m, k} S_{k n}^{-1}\left(V_{0}\right) \exp \left(-i E_{k} t\right) S_{m k}\left(V_{0}\right) C_{m}(0)
$$

where $\varepsilon_{k}\left(V_{0}\right)$ are eigenenergies of the Hamiltonian $H_{0}+$ $H_{1}\left(V_{0}\right)$ and $S_{n m}\left(V_{0}\right)$ are the unitary matrices transforming from the noninteracting eigenbasis (the one corresponding to $H_{0}$ ) to the eigenbasis of the full Hamiltonian $H_{0}+H_{1}$. It is implied in Eq. (14) that at a fixed value of $V_{0}$, the time evolution is performed as the interplay between the three different eigenenergies. This is sufficient evidence that if the eigenenergies are appropriately adjusted, the population of the auxiliary state 


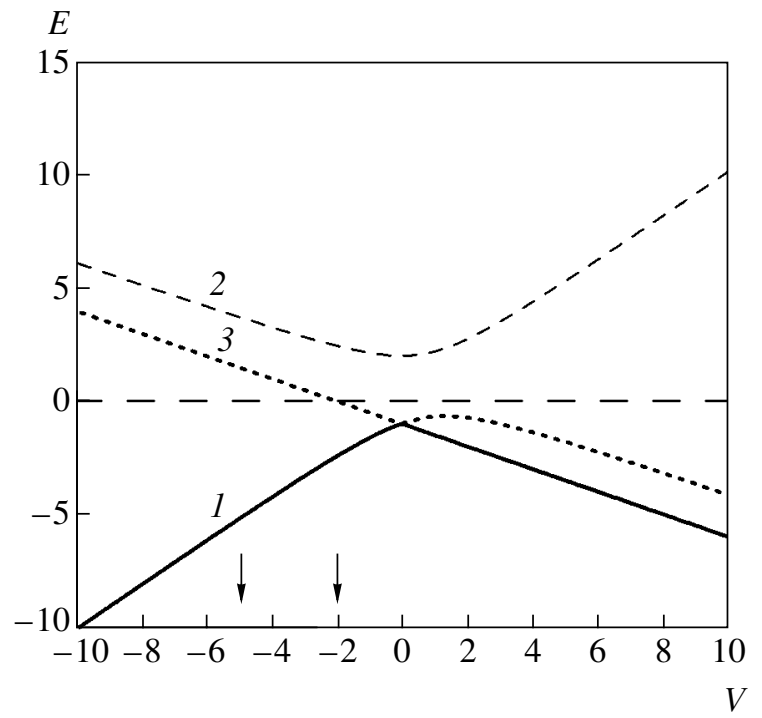

Fig. 8. Energy versus electrostatic potential. Curves 1 and 3 (solid and dotted lines) are the energies that become degenerate at $V_{0}=0$, and curve 2 (the dashed line) is the energy of the auxiliary control state $|c\rangle$. The arrows indicate the values of the potential $V_{0}$ corresponding to the operational points of the bit-flip and Hadamard gates.

(in the angular-momentum basis) can vanish for certain initial conditions. At these time instants, the three-state system instantaneously collapses into the qubit subspace without loss of any information if the auxiliary state $|c\rangle$ was initially unoccupied. A necessary condition for the instantaneous collapse into the qubit subspace (i.e., the degenerate-level subspace) is a commensuration condition between the eigenenergies $\varepsilon_{k}\left(V_{0}\right), k=1,2,3$ such that the exponential factors in Eq. (14) destructively interfere at fixed tune instants to destroy the nondiagonal correlations. The required commensuration can be expressed by the condition

$$
\varepsilon_{3}-\varepsilon_{1}=v\left(\varepsilon_{2}-\varepsilon_{3}\right)
$$

for integer $v$. Equation (15) guarantees periodic collapses of the wavefunction onto the desired basis, and the next step is to find whether the desired qugate operations can be realized simultaneously in this basis. For the corresponding values of the potential respecting Eq. (15), we find

$$
V_{0}(v)=-\frac{2}{3 v}\left[v^{2}+v+1+(v-1) \sqrt{v^{2}+4 v+1}\right] .
$$

In particular, we note that for $v=1$, we have $V_{0}^{(1)}=-2$ and at $v=3$, we have

$$
V_{0}^{(3)}=-\frac{2}{9}(13+2 \sqrt{22})=-4.9735,
$$

and we succeeded in finding two qugates in our first few attempts. As shown below, these two cases yield the bitflip and Hadamard transformations of the qubit [17].
The $v=1$ case can be explicitly proved by verifying the identity

$$
\begin{gathered}
\exp \left\{-i t\left(\begin{array}{ccc}
-1 & -1 & -1 \\
-1 & 2 & -1 \\
-1 & -1 & -1
\end{array}\right)\right\} \\
=\frac{1}{2}\left(\begin{array}{ccc}
1+c+s & s & -1+c+s \\
s & 2(c-s) & s \\
-1+c+s & s & 1+c+s
\end{array}\right),
\end{gathered}
$$

where

$$
c=\cos (t \sqrt{6}), \quad s=i \sqrt{\frac{2}{3}} \sin (t \sqrt{6}) .
$$

At $s=0$ (i.e., $c= \pm 1$ ), the transformation matrix of qubit states is block-diagonalized in the subspace of states 1 , 3 (i.e., the qubit states $|0\rangle,|1\rangle$ ) and the upper state 2 (i.e., the auxiliary "control" state $|c\rangle)$. In particular, for $c=$ -1 , the bit-flip is performed between the qubit states.

In Fig. 9, the populations $p_{n}(t)=\left|C_{n}(t)\right|^{2}$ of the states are plotted for the mentioned cases $v=1$ and $v=3$. The instantaneous collapse to the qubit subspace is obtained at $t=t_{1}$ for $\mathrm{v}=1$ and at $t=t_{3}$ for $\mathrm{v}=3$ if the auxiliary level is unoccupied at $t=0$. We found these critical times as (in units of $\hbar / \tau$ )

$$
\begin{gathered}
t_{1}=\frac{\pi}{\sqrt{6}}=1.2825, \\
t_{3}=\frac{\pi}{2\left[E_{2}\left(V_{0}\right)-E_{3}\left(V_{0}\right)\right]_{v=3}}=0.7043,
\end{gathered}
$$

where the eigenenergies are

$$
\begin{gathered}
E_{1,3}\left(V_{0}\right)=\frac{1+V_{0} / 2}{2} \mp \frac{3}{2} \sqrt{1-\frac{V_{0}}{2}+\frac{V_{0}^{2}}{4}}, \\
E_{2}\left(V_{0}\right)=-1-\frac{V_{0}}{2}
\end{gathered}
$$

for $V_{0} \leq 0$. We note that the configuration $\left(t_{1}, v=1\right)$ performs the bit-flip $|0\rangle \longleftrightarrow|1\rangle$, whereas $\left(t_{3}, v=3\right)$ creates the equally populated Hadamard-like superpositions of $|0\rangle$ and $|1\rangle$. These operations are represented in the qubit subspace by the matrices (overall phases are not shown)

$$
G_{1}=\left(\begin{array}{ll}
0 & 1 \\
1 & 0
\end{array}\right) \text { and } G_{3}=\frac{1}{\sqrt{2}}\left(\begin{array}{cc}
1 & -i \\
-i & 1
\end{array}\right) \text {. }
$$

The dotted lines show the time dependence of the auxiliary population. The arrows indicate the "operational 
point" of the qugate, the time of evolution corresponding to the return to the invariant qubit. The $G_{1}$ transformation manifests the bit-flip (NOT gate) and $G_{3}$ is similar to the Hadamard gate [17] except for the phase shift $\pi / 2$.

\section{QUANTUM BISTABILITY AND SPONTANEOUS CURRENTS} IN A COUPLED ELECTRON-PHONON SYSTEM

In the tight-binding approximation, the Hamiltonian of the loop in the secondary quantized form is given by

$$
\begin{gathered}
H=\sum_{j=1}^{N}\left(\tau_{j} a_{j \sigma}^{+} a_{j+1, \sigma} e^{i \alpha_{j}}+\text { H.c. }\right)+U \sum_{j=1}^{N} n_{j \uparrow} n_{j \downarrow} \\
+V \sum_{j=1, \sigma, \sigma^{\prime}}^{N} n_{j \sigma^{\prime}} n_{j+1, \sigma^{\prime}} \\
+\frac{1}{2} W \sum_{j=1}^{N}\left(\theta_{j}-\theta_{j}^{0}\right)^{2}+\frac{1}{2} K \sum_{j=1}^{N}\left(\theta_{j}-\theta_{j+1}\right)^{2}
\end{gathered}
$$

where $\tau_{j}$ is the hopping amplitude between two adjacent configurational sites, $j$ and $j+1$,

$$
\tau_{j}=\tau_{0}+g\left(\theta_{j}-\theta_{j+1}\right), \quad n_{i \sigma}=a_{i \sigma}^{+} a_{i \sigma},
$$

and

$$
\alpha_{j}=\frac{2 \pi f}{N}+\left(\theta_{j}-\theta_{j+1}\right) f
$$

is the Aharonov-Bohm phase (a Peierls substitution for the phase of hopping amplitude). Next, $a_{j \sigma}^{+}$is the creation (and $a_{j \sigma}$ is the annihilation) operator of the electron at site $j$ with spin $\sigma ; \theta_{j}, j=1,2, \ldots, N$ are the angles of distortion of site locations from their equilibrium positions $\theta_{j}^{0}=2 \pi j / N$ satisfying the requirement

$$
\sum_{j=1}^{N} \theta_{j}=0 ;
$$

and $g$ is the electron-phonon coupling constant. The interaction in Eq. (22) reflects the property that the hopping amplitude depends on the distance between the localization positions and assumes that the displacement $\theta_{j}-\theta_{j+1}$ is small in comparison to $2 \pi / N$. $U$ and $V$ are Hubbard parameters of the on-site and intrasite interactions. $W$ is the binding energy of the loop to external environment (a substrate) such that the loop
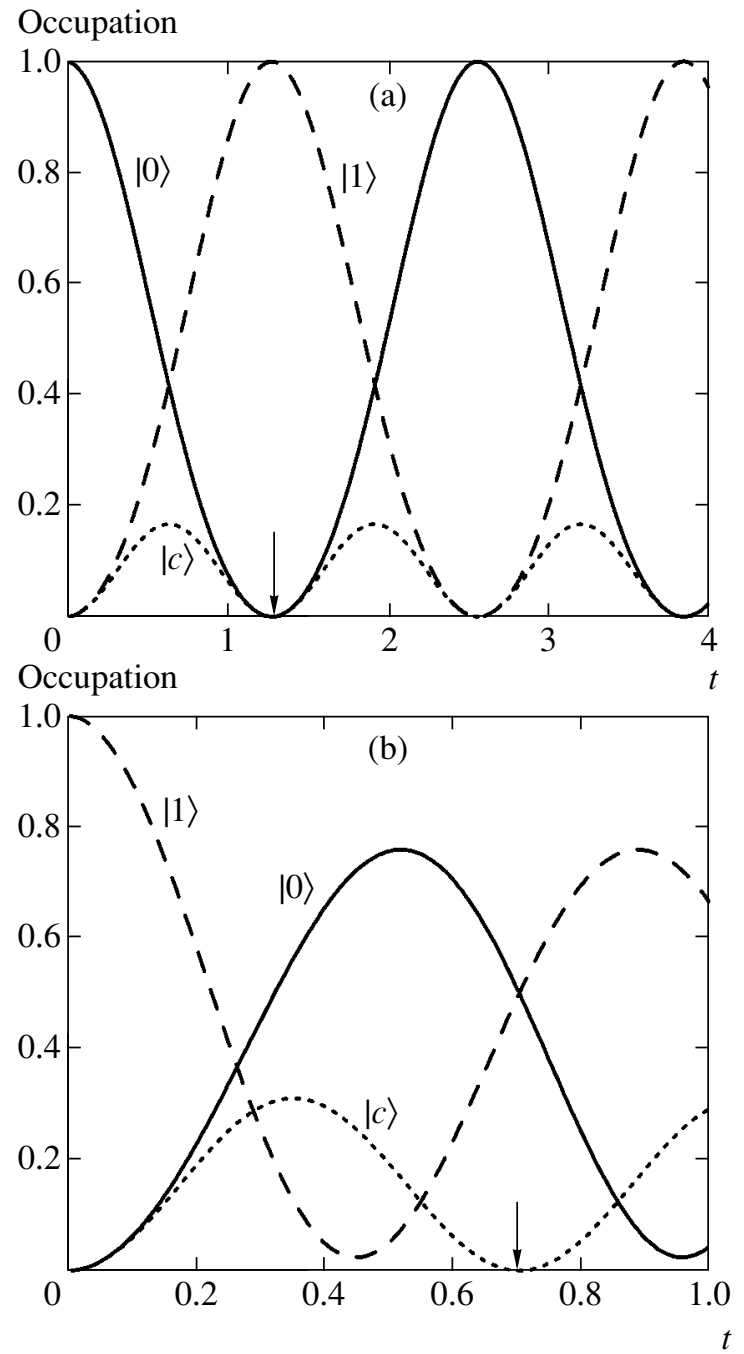

Fig. 9. Evolution diagrams of the quantum gate $G_{1}$ (a) and $G_{3}$ (b). Solid and dashed lines are the time dependences of the population of states $|0\rangle$ and $|1\rangle$. The dotted line shows the time dependence of the auxiliary-state population. The arrow indicates the "operational point" of the qugate, i.e., the evolution time corresponding to the return to the invariant qubit subspace.

passes into the azimuthally symmetric configuration $\theta_{i}=\theta_{i}^{0}$ as $W \longrightarrow \infty$.

The parameters are assumed such that the system is not superconductive (e.g., $U>0$; anyway, the superconductivity is not allowed for a $1 \mathrm{D}$-system and it is forbidden for a small system). The last term in Hamiltonian (21) is the elastic energy and $K$ is the stiffness parameter of the lattice.

In the smallest loop, the one with three sites $(N=3)$, only two free parameters of the lattice displacement, $X_{1}$ and $X_{2}$, remain:

$$
\theta_{1}=X_{1}+X_{2}, \quad \theta_{2}=-X_{1}+X_{2}, \quad \theta_{3}=-2 X_{2},
$$




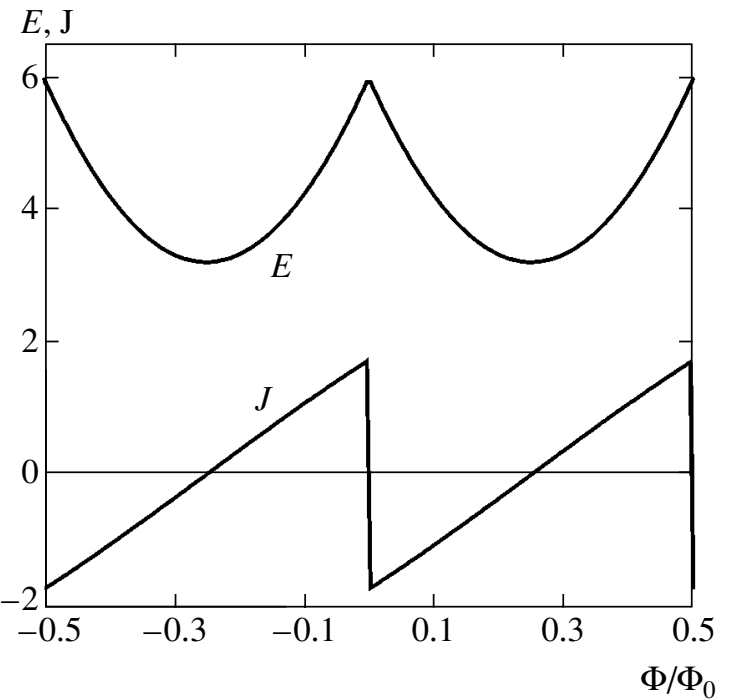

Fig. 10. Lower curve: current versus magnetic flux in a 3 -site loop with 3 noninteracting electrons. Upper curve: energy versus flux in the loop. The hopping parameter is $\tau_{0}=-1$. The energy is reset and arbitrarily shifted upward for clarity.

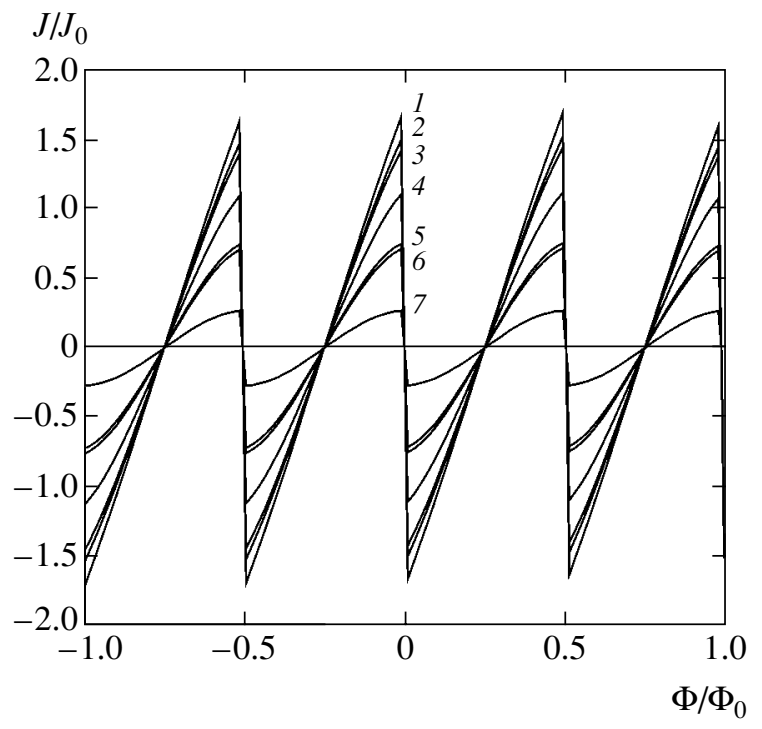

Fig. 11. Spontaneous persistent current versus flux for $\tau_{0}=$ -1 and various values of the Hubbard parameter $U: U=0$ (1), -2 (2), 2 (3), -5 (4), 5 (5), -10 (6), 10 (7).

which are decomposed with respect to secondary quantized Bose operators $b_{1}$ and $b_{2}$ as

$$
\begin{aligned}
& X_{1}=\left(\frac{3 K}{\omega}\right)^{1 / 4}\left(b_{1}+b_{1}^{+}\right), \\
& X_{2}=3\left(\frac{K}{3 \omega}\right)^{1 / 4}\left(b_{2}+b_{2}^{+}\right) .
\end{aligned}
$$

System (21) is solved numerically with the ABC compiler [41], which includes the creation-annihilation operators as its parameter types. These are generated as compiler macros with sparse matrices

$$
\begin{gathered}
A_{n}=C_{n}^{\left(N_{1}\right)} \otimes 1^{\left(N_{2}\right)}, \quad \text { fermionic sector, } \\
B_{n}=1^{\left(N_{1}\right)} \otimes C_{n}^{\left(N_{2}\right)}, \quad \text { bosonic sector, }
\end{gathered}
$$

where $1^{(N)}$ is the unit matrix of size $2^{N} ; C_{n}^{(N)}, n=1, \ldots$, $N$ are Fermi/Bose operators in a space of the same dimension,

$$
C_{n}^{(N)}=(u \otimes)^{N-n} a(\otimes V)^{n-1} ;
$$

and $a, u$, and $v$ are the $2 \times 2$ matrices (with $\otimes$ being the symbol of the Kronecker matrix product):

$$
\begin{gathered}
a=\left(\begin{array}{ll}
0 & 0 \\
1 & 0
\end{array}\right), \\
u=\left(\begin{array}{ll}
1 & 0 \\
0 & 1
\end{array}\right), \quad v=\left(\begin{array}{ll}
1 & 0 \\
0 & \eta
\end{array}\right),
\end{gathered}
$$

and

$$
\eta= \begin{cases}-1, & \text { fermionic sector } \\ 1, & \text { bosonic sector. }\end{cases}
$$

The bosons are considered hardcore bosons, such that there are only two discrete states for each mode of displacement. We calculate the ground state of Hamiltonian (21) as a function of the magnetic flux $f$ (a classical variable). In application to real atomic (macromolecular) systems, we can consider $X_{1}$ and $X_{2}$ as classical variables because quantum uncertainties in the coordinates $\left(\Delta X_{1,2} \sim(\hbar / M \omega)^{1 / 2}\right)$ are typically much smaller than the interatomic distances ( $M$ is the mass of an atom and $\omega \sim 10^{13} \mathrm{~s}^{-1}$ is the characteristic vibration frequency). The energy of the loop is calculated as a function of $X_{1}$ and $X_{2}$ and further minimized with respect to $X_{1}$ and $X_{2}$ for each value of $f$. The nonzero values of $X_{1}$ and $X_{2}$ signify a "lattice" (the ionic core of the macromolecule) instability against the structural transformation, analogous to the Peierls transition.

In the noninteracting system $(U, V, W, g=0)$, the energy versus the flux $f$ shows a kink with a maximum at $f=0$ (Fig. 10) in the half-filling case, i.e., at a number of electrons $n$ equal to the number of sites $N$, as well as in a broader range of values of $n$ at larger $N$. Actually, as is clear from Fig. 4, such a dependence is typical of any $N \geq 3$ system for a number of (fixed) values of $n$.

The 3-site loop's $E(f)$ dependence is shown in Fig. 10 together with the dependence of the current on $f$. The latter shows a discontinuity at $f=0$ of the same order of magnitude as the standard value of the 
persistent current. The current at $f=0$ is paramagnetic because the energy vs. flux has a maximum rather than a minimum at $f=0$. The on-site interaction reduces the persistent current amplitude near zero flux (Fig. 11) but does not remove its discontinuity at $f=0$. Therefore, the strongest opponent of the Aharonov-Bohm effect, the electron-electron interaction, leaves the current qualitatively unchanged.

On the other hand, the electron-phonon interaction (considered here classically in regard to lattice vibration) flattens the $E(f)$ dependence near the peak value (see Fig. 12a). At large stiffnesses $K$, this flattening remains important only for small magnetic fluxes, much smaller than the flux quantization period $\Delta \Phi=$ $\Phi_{0}$. We note that the persistent current peak reduces in its amplitude only slightly near $\Phi=0$. As is seen from Fig. 12b, the electron-phonon interaction splits the singularity at $\Phi=0$ to two singularities at $\Phi= \pm \Phi_{\text {sing }}$. Outside the interval $-\Phi_{\text {sing }}<\Phi<\Phi_{\text {sing }}$, the structural transformation is blocked by the Aharonov-Bohm flux. The range of magnetic fluxes between $-\Phi_{\text {sing }}$ and $\Phi_{\text {sing }}$ determines the domain of the developing lattice transformation, which signifies itself with nonzero values of lattice deformations $X_{1}$ and $X_{2}$. This property allows us to suggest that the spontaneous persistent current state (a peak of dissipationless charge transport at or near the zero flux) remains at a nonzero $\Phi$ when the electronphonon coupling is not too strong or when the lattice stiffness is larger than a certain critical value.

\section{DISCUSSION}

In conclusion, we considered the Aharonov-Bohm effect in an angular-periodic macromolecular structure, like that of an aromatic cyclic molecule, and established the existence of a persistent current and also a spontaneous current when the Aharonov-Bohm flux is not applied to the ring. Strong coupling of electron hopping to the ion core of the molecule removes the spontaneous current, which is nevertheless restored at a (small) magnetic field, or when the loop has large stiffness or is strongly bound to an external azimuthal-periodic environment (a substrate). Degenerate states of the loop at $\Phi=\Phi_{0} / 2$ and at $\Phi=0$ may serve as components of a qubit that are operated by static voltages applied in the plane of the loop perpendicular to the direction of the Aharonov-Bohm flux.

The papers of Gatteschi et al. [18, 19] are particularly noteworthy, in which an azimuthal-periodic molecular structure (a "ferric wheel" $\left.\left[\mathrm{Fe}(\mathrm{OMe})_{2}\left(\mathrm{O}_{2} \mathrm{CCH}_{2} \mathrm{Cl}\right)\right]_{10}\right)$ exhibited periodic variation of its magnetization as a function of the magnetic flux; we assume that the periodicity with large period can be attributed to persistent currents. The above macromolecular structure is more complex than the one we considered because it contains magnetic ions with strong
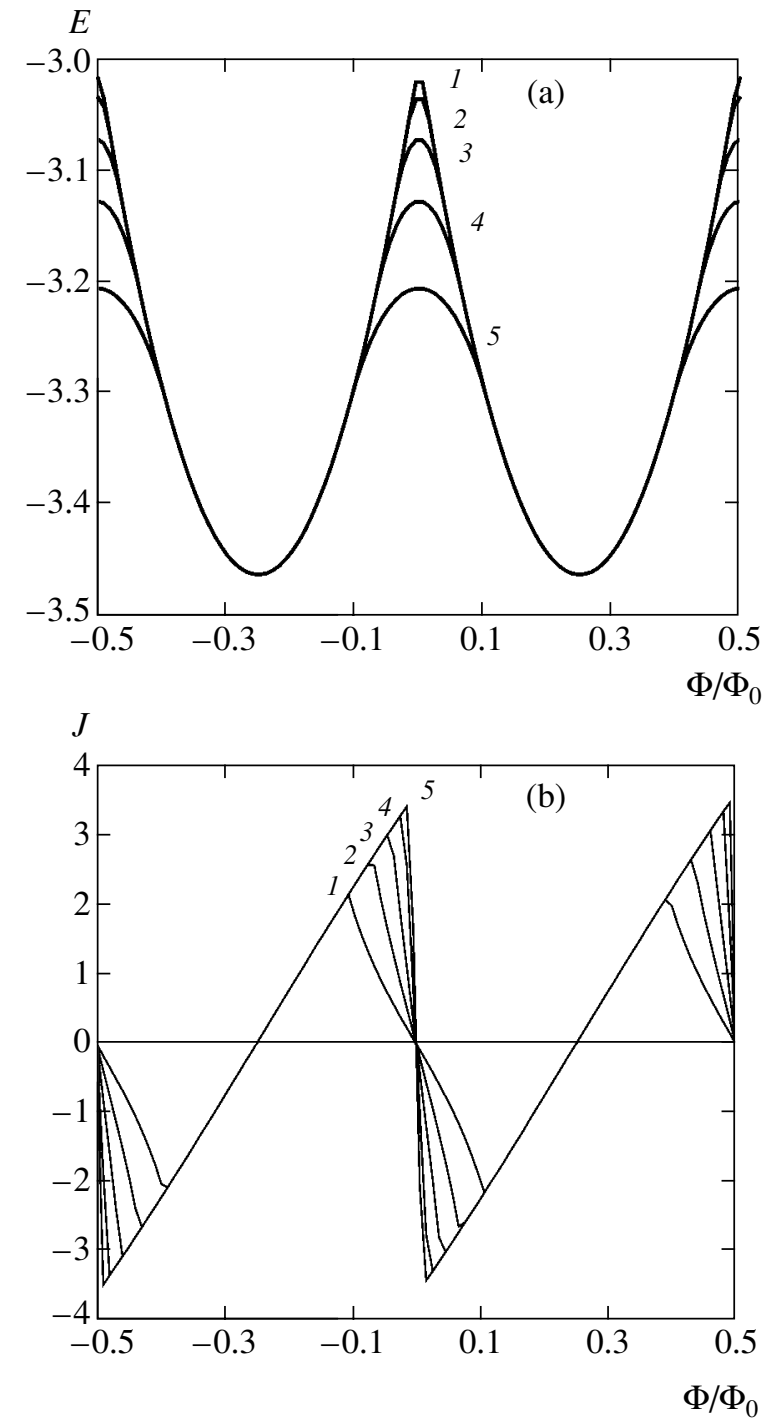

Fig. 12. Energy (a) and current (b) versus flux in a loop of noninteracting electrons coupled to the lattice with the coupling parameter $g=1$ and various values of the stiffness parameter $K: K=2(1), 3(2), 5(3), 10(4), 20(5)$.

exchange interactions such that the actual magnetic field in the ring may be larger than the externally applied field. If this suggestion proves correct, it will open the possibility of engineering macromolecular structures (qubits and qugates) based on the AharonovBohm effect, for purposes of quantum computation. Apart from this, the very existence of a nonzero nondecaying current in a nonsuperconductive system is, in our opinion, of fundamental physical interest.

\section{ACKNOWLEDGMENTS}

I would like to thank Prof. D. Averin for helpful discussions and advice, and Prof. K. Likharev for comments on quantum computational aspects of nanoscale physics. 


\section{REFERENCES}

1. F. Bloch, Phys. Rev. B 2, 109 (1970).

2. I. O. Kulik, Pis'ma Zh. Éksp. Teor. Fiz. 11, 407 (1970) [JETP Lett. 11, 275 (1970)].

3. M. Büttiker, Y. Imry, and R. Landauer, Phys. Lett. 96A, 365 (1983)

4. Y. Aharonov and D. Bohm, Phys. Rev. 115, 485 (1959).

5. I. O. Kulik, in Quantum Mesoscopic Phenomena and Mesoscopic Devices in Microelectronics, Ed. by I. O. Kulik and R. Ellialtioglu (Kluwer Academic, Dordrecht, 2000), p. 259.

6. E. Teller, Z. Phys. 67, 311 (1931).

7. L. D. Landau, Z. Phys. 64, 629 (1930).

8. N. B. Brandt, E. N. Bogachek, D. V. Gitsu, et al., Fiz. Nizk. Temp. 8, 718 (1982) [Sov. J. Low Temp. Phys. 8, 358 (1982)].

9. L. P. Levy, G. Dolan, J. Dunsmuir, and H. Bouchiat, Phys. Rev. Lett. 64, 2074 (1990).

10. V. Chandrasekhar, R. A. Webb, M. J. Brady, et al., Phys. Rev. Lett. 67, 3578 (1991).

11. D. Mailly, C. Chapelier, and A. Benoit, Phys. Rev. Lett. 70, 2020 (1993).

12. C. Schönenberger, A. Bachtold, C. Strunk, et al., Appl. Phys. A 69, 283 (1999).

13. B. L. Altshuler, A. G. Aronov, and B. Z. Spivak, Pis'ma Zh. Éksp. Teor. Fiz. 33, 101 (1981) [JETP Lett. 33, 94 (1981)].

14. A. Barone, T. Hakioglu, and I. O. Kulik, condmat/0203038.

15. I. O. Kulik, T. Hakioglu, and A. Barone, Eur. Phys. J. B 30, 219 (2002).

16. I. O. Kulik, in Towards the Controllable Quantum States: Mesoscopic Superconductivity and Spintronics, Ed. by H. Takayanagi and J. Nitta (World Sci., River Edge, N.J., 2003), p. 302.

17. M. A. Nielsen and I. L. Chuang, Quantum Computation and Quantum Information (Cambridge Univ. Press, Cambridge, 2000).

18. D. Gatteschi, A. Caneschi, L. Pardi, and R. Sessoli, Science 265, 1054 (1994).

19. K. L. Taft, C. D. Delfs, G. C. Papaefthymiou, et al., J. Am. Chem. Soc. 116, 823 (1994).

20. H. F. Cheung, E. K. Riedel, and Y. Gefen, Phys. Rev. Lett. 62, 587 (1989).

21. I. O. Kulik and I. K. Yanson, The Josephson Effect in Superconductive Tunneling Structures (Nauka, Moscow,
1970; Israel Program for Scientific Translations, Jerusalem, 1972).

22. A. Barone and G. Paterno, Physics and Applications of the Josephson Effect (Wiley, New York, 1982; Mir, Moscow, 1984).

23. A. J. Leggett, in Chance and Matter, Ed. by J. Souletier, J. Vannimenus, and R. Stora (Elsevier, Amsterdam, 1996), p. 395.

24. K. K. Likharev, Dynamics of Josephson Junctions and Circuits (Gordon and Breach, Amsterdam, 1996).

25. Y. Makhlin, G. Schön, and A. Schnirman, Rev. Mod. Phys. 73, 357 (2001).

26. D. V. Averin, Fortschr. Phys. 48, 1055 (2000).

27. H.-F. Cheung, Y. Gefen, E. K. Riedel, and W.-H. Shih, Phys. Rev. B 37, 6050 (1988).

28. S. Latil, S. Roche, and A. Rubio, Phys. Rev. B 67, 165420 (2003).

29. I. O. Kulik, A. S. Rozhavskii, and E. N. Bogachek, Pis'ma Zh. Éksp. Teor. Fiz. 47, 251 (1988) [JETP Lett. 47, 303 (1988)].

30. M. I. Vischer, B. Rejaei, and G. E. W. Bauer, Europhys. Lett. 36, 613 (1996).

31. Yu. I. Latyshev, O. Laborde, P. Monceau, and S. Klaumünzer, Phys. Rev. Lett. 78, 919 (1997).

32. J. Yi, M. Y. Choi, K. Park, and E.-H. Lee, Phys. Rev. Lett. 78, 3523 (1997).

33. B. Nathanson, O. Entin-Wohlman, and B. Mülschlegel, Phys. Rev. Lett. 80, 3416 (1998).

34. G. Montambaux, Phys. Rev. Lett. 80, 3417 (1998).

35. R. E. Peierls, Quantum Theory of Solids (Clarendon, Oxford, 1955; Inostrannaya Literatura, Moscow, 1956).

36. H. J. Jahn and E. Teller, Proc. R. Soc. London, Ser. A 161, 220 (1937).

37. W. A. Harrison, Electronic Structure and the Properties of Solids (Cambridge Univ. Press, Cambridge, 1972; Mir, Moscow, 1983).

38. J. M. Ziman, Principles of the Theory of Solids, 2nd ed. (Cambridge Univ. Press, London, 1972; Mir, Moscow, 1966).

39. C. Kittel, Introduction to Solid State Physics, 7th ed. (Wiley, New York, 1996; Nauka, Moscow, 1978).

40. L. N. Bulaevskii, Usp. Fiz. Nauk 115, 263 (1975) [Sov. Phys. Usp. 18, 131 (1975)].

41. I. O. Kulik, in Technical Proceedings of the 2003 Nanotechnology Conference and Trade Show, Ed. by M. Laudon and B. Romanowicz (Computational, Boston, 2003), Vol. 2, p. 531. 